\title{
Optimization of Forensic Analyses with Latest Generation EDS and EBSD on Multi-Function SEMs
}

\author{
Matt Hiscock ${ }^{1}$ \\ 1. Oxford Instruments NanoAnalysis, High Wycombe, UK
}

Laboratories offering forensic services often have to deal with high levels of demand for a variety of different analyses across multiple techniques. This means that there is a constant requirement that instrument time is used as efficiently as possible. In order to maximize this, the analyses themselves must be conducted over as short a time as possible but still at the highest quality levels (the consequences of an incorrect analysis could be a person's freedom). In order to fully meet all of these criteria, a number of requirements are put onto the measurement chain. We will discuss these, and their application to forensic analyses here.

One of the most well known forensic analyses conducted with the scanning electron microscope (SEM) is the search for and classification of gunshot residue (GSR) particles. GSR particles are searched for by imaging the entirety of an SEM stub upon which the particles have been collected and acquiring Energy Dispersive X-ray Spectrometry (EDS) spectra from any candidate particles (e.g. Fig 1A). This process is carried out automatically with the user manually reviewing the analyzed particles to confirm that they are GSR and acquiring additional, high resolution data for presentation to the court. It is possible for this work to be quite time consuming both due to the need to acquire sufficient EDS data to be statistically certain of composition and because imaging must be performed at sufficiently high magnification and resolution to directly image candidate GSR particles which could be on a scale of fractions of microns. EDS acquisition times have the potential to form a very significant proportion of run durations, particularly for highly loaded samples. If a fixed number of x-ray counts is defined as being required from each candidate particle in order to make a high quality measure of composition (e.g. Fig 1B), then only the speed of acquisition of that data can be adjusted to decrease overall run duration. By utilizing the latest generation of EDS detectors (Oxford Instruments' Ultim Max), very high count rates can be achieved at the level of accuracy needed for this application, allowing both throughput and accuracy requirements to be met. The use of these detectors in forensics will be discussed. In the case of a GSR sample stub not containing many particles in each field of view, the time taken for image acquisition may form a significant proportion of the run time. In order to alleviate this, advanced imaging strategies can be employed so that fast dwell times are used for complete fields, with slow dwell times only utilized at particle locations. In addition, we consider the use of multiple detectors to aid in the identification of the brand of ammunition used in shootings. It is only by combining both high performance detectors with software that has been specifically tuned to the application in question that the high demands of this and other forensic applications can be achieved.

We also consider the application of a second SEM based analytical technique, Electron Backscatter Diffraction (EBSD), to the forensic sciences. Here, recent developments have allowed an order of magnitude improvement in performance and greatly enhanced flexibility which has important ramifications for the applicability of EBSD to routine forensic problems. We consider this in terms of a case study: The reconstruction of a serial number from a metallic object which a suspect had attempted to destroy. 
When a metal object is imprinted with a serial number, damage is induced in the crystal structure of that metal to a depth greater than the deepest point of that imprint. When a person who is trying to destroy lines of evidence removes one of these serial numbers they do so only to the point where it is no longer visible by eye. As an example of this, we studied a key which had had the serial number scratched off (Figure 2A). The key was polished to the point where it would be acceptable for analysis by EBSD with (Oxford Instruments' Symmetry CMOS EBSD detector) - at this point no remnants of the original serial number were visible, even under electron imaging in the SEM. In order to reveal the serial number, the sample was mapped with EBSD and an image showing the quality of the diffraction pattern acquired at each point was created - a band contrast image. In this image lighter pixels relate to less deformed areas and darker pixels to more deformed areas. It is clear to see that the serial number is easily readable in this image (Figure 2A).

We demonstrate here that recent advancements in hardware and software have real implications for forensic analyses, allowing greater flexibility, sensitivity and throughput to ensure that this important work is completed as efficiently and accurately as possible.

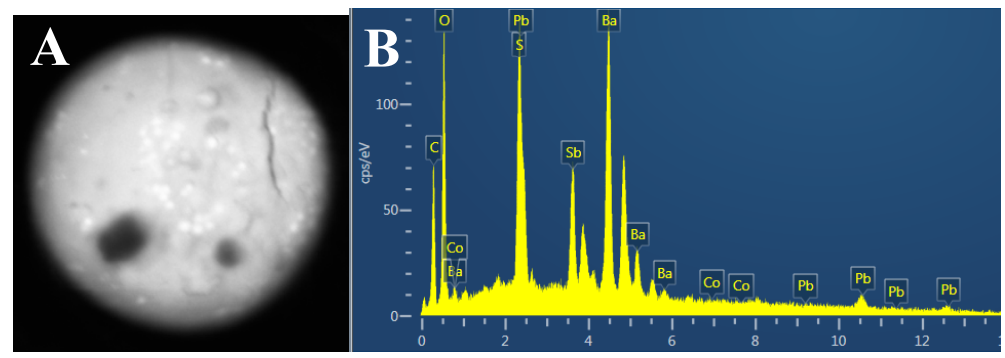

Figure 1. Example image of a GSR particle and spectrum acquired from that particle - spectrum has a sufficiently high number of x-ray counts in it for accurate element ID and peak overlap deconvolution. With very sensitive, large area detectors this spectrum can be acquired with very short live times of fractions of a second.

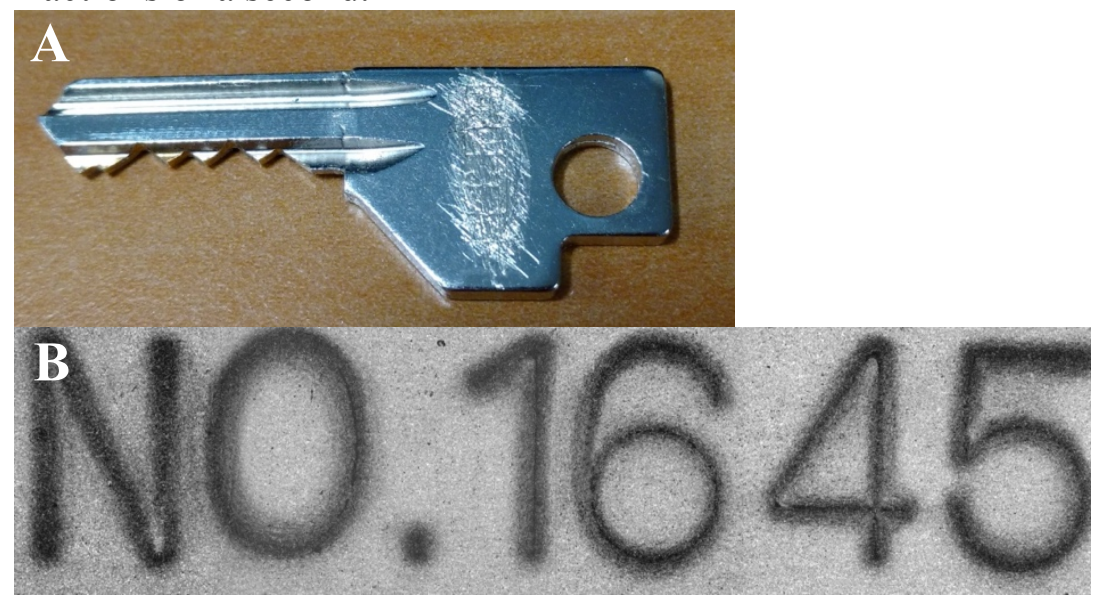

Figure 2. Reconstruction of a scratched off serial number with EBSD. The deformation induced by the imprinting of the serial number extends deeper into the metal of the key than is visible. As such, attempting to scratch it off does not remove all traces of the serial number and it can be recovered. 